\title{
Simulation and parameterisation of spallation neutron distributions
}

\author{
Asiya Rummana* \\ International Institute for Accelerator Applications, \\ University of Huddersfield, UK \\ E-mail: Asiya.Rummana@hud.ac.uk

\section{Roger John Barlow} \\ International Institute for Accelerator Applications, \\ University of Huddersfield, UK \\ E-mail: R.Barlow@hud.ac.uk
}

\begin{abstract}
We aim to produce a simple model to predict the numbers and properties of spallation neutrons. We chose a lead target of close to optimal dimension that is $30 \mathrm{~cm}$ long and $30 \mathrm{~cm}$ in radius and performed simulations of neutrons produced by a beam of proton incident on the target. The beam energy ranges from $100 \mathrm{MeV}$ to $1400 \mathrm{MeV}$. We chose two programs MCNPX and Geant4 and compared the results against each other. The investigations shows good agreement between the two programs for the overall numbers, energy spectra, spatial and radial distribution. Finally we produced a parametrized form which can be used for the neutronics studies in the reactor cores.
\end{abstract}

4th Workshop on ADS and thorium

31 August - 2 September 2016

University of Huddersfield, England

\footnotetext{
* Speaker.
} 


\section{INTRODUCTION}

Spallation process being an established technique for the production of high intensity neutron flux, the design of target and neutronic studies is an area of growing interest. The present study covers the detailed investigations performed for the neutron distribution for ADSR. For spallation neutron source, proton beam ranging from $100 \mathrm{MeV}$ to $1.4 \mathrm{GeV}$ is incident on a lead target that is a cylinder with typical dimensions as $30 \mathrm{~cm}$ in radius and $30 \mathrm{~cm}$ in length [1]. The detectors are placed at various locations around the target to record the neutron distributions from near end (where the proton beam enters), far end (opposite to near face) and the sides of the cylindrical target. The investigations are performed for the overall number of neutrons produced, their longitudinal distribution, radial distribution, energy spectra and their cross section in lead.
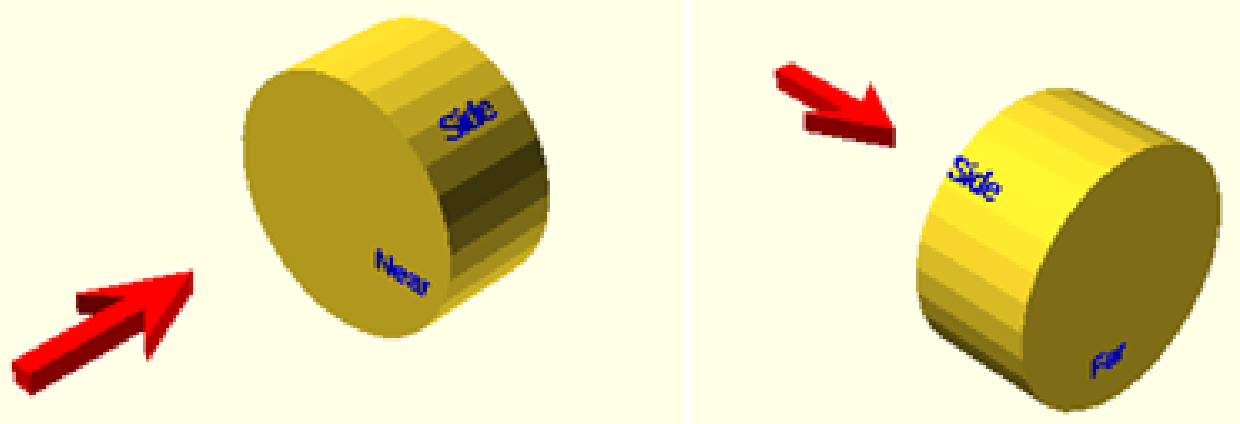

Figure 1: Schematic view of target

In the present study, computations are performed using GEANT4 [2] simulation code. As a benchmarking process, these results are compared with the corresponding MCNPX [3] predictions which was used with default ENDF/B-V library. In the preliminary studies [4, 5] Geant4 simulations are performed initially with Bertini model and then with its high precision version but both of these show serious discrepancies with MCNPX predictions. Binary cascade model with high precision BIC_HP gives good agreement with the MCNPX results and this model is used for the results presented here. It is found that the properties of spallation neutrons can be parametrized by simple forms which can then be used as a source term for neutronics simulations for ADSR reactors [6].

\section{NEUTRON NUMBERS}

Overall numbers of spallation neutrons using Geant4 and MCNPX are shown in Fig 2. They are in good agreement for the total numbers however small difference in the relative importance of sides and ends. Both the codes predict that more neutrons are available at near face where 
the proton beam falls. This is somewhat counter-intuitive, but can be explained as the neutrons undergo many collisions in lead and 'forget' their original direction, and the near face receives the full energy of proton beam.

As the proton energy increases the number of neutron also increase. At $1 \mathrm{GeV}$ approximately 30 neutrons are available per proton. The shape is very roughly linear and above $600 \mathrm{MeV}$ the concave becomes convex. So it can be nicely fitted through two parabolas above and below $600 \mathrm{MeV}$.
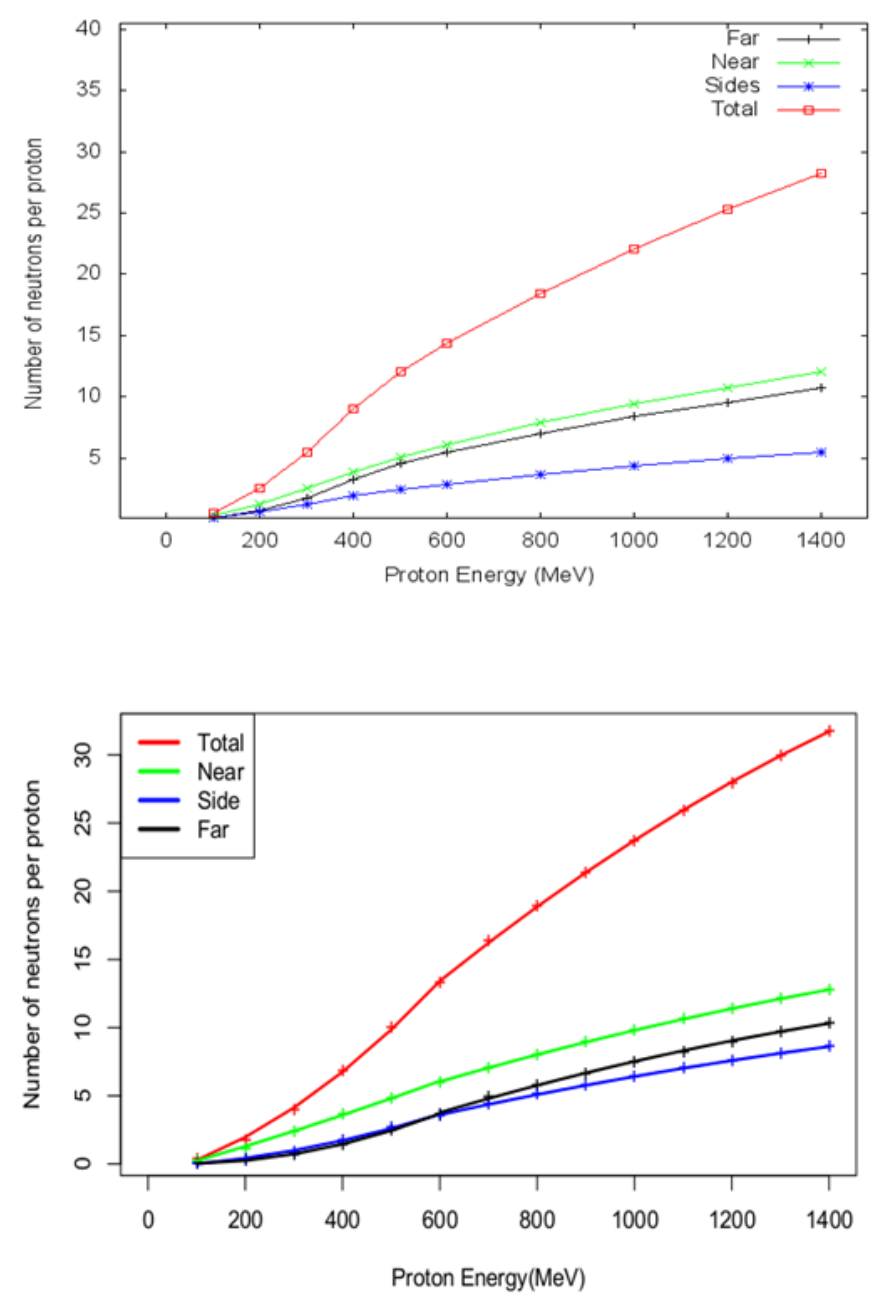

Figure 2: Number of neutrons from spallation target for Geant4 (Top) and MCNPX (Bottom)

\section{ENERGY DISTRIBUTIONS}

The neutron energy spectra can be categorized as high energy spectra, low energy spectra and intermediate energy spectra showing different characteristics.

\subsection{High energy neutrons}

In the high energy spectrum the effects of spallation appear in a tail of neutrons which falls 
roughly exponentially with energy, with an additional cutoff as the energy of the original proton is reached. The predictions of energy spectra for these two categories by Geant4 and MCNPX are shown in Fig 3. Spectra are plotted for $1000 \mathrm{MeV}$ beam energy. Both the codes predicts that the high energy component of spectra are dependent on proton energy and the contribution from different faces are also different.

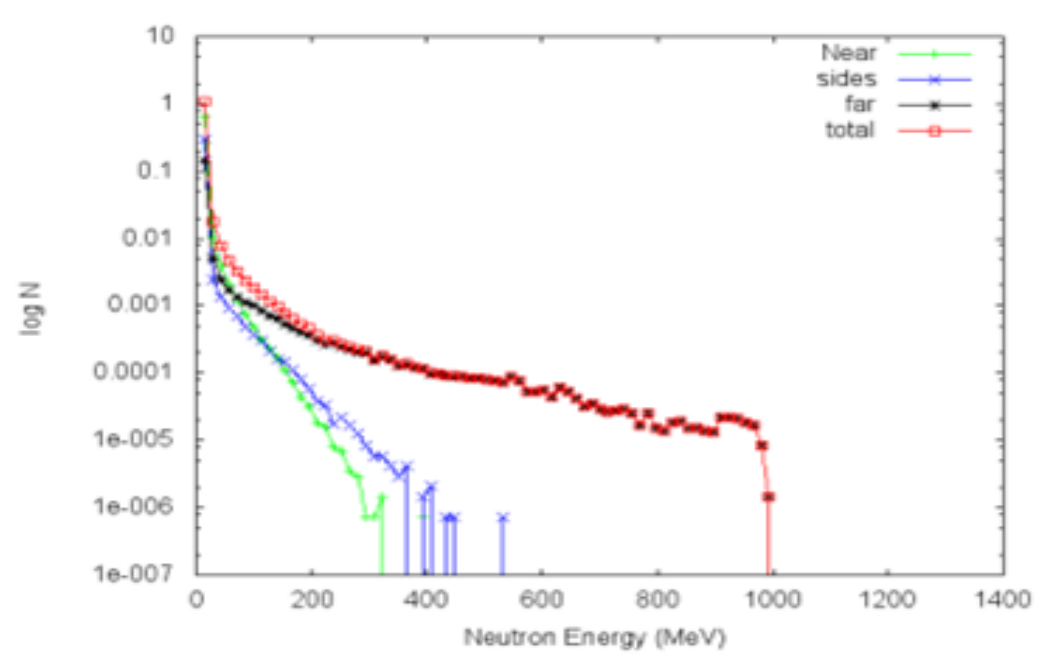

Proton energy $1000 \mathrm{MeV}$

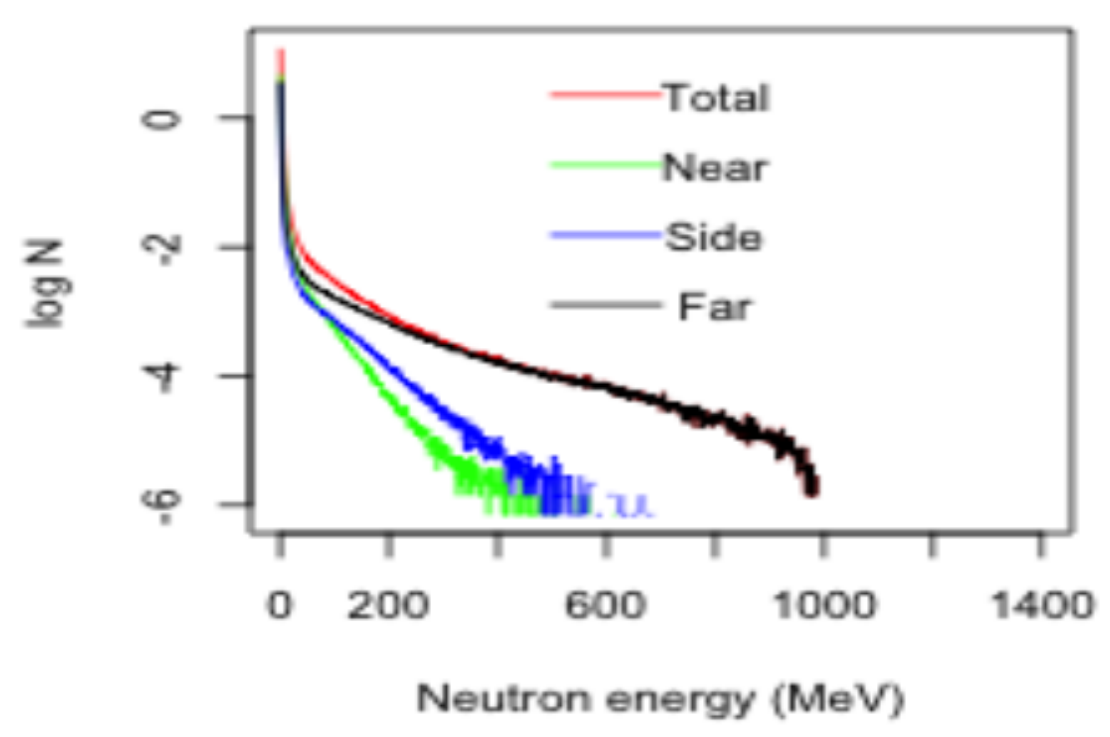

Figure 3: High energy spectra Geant4 (Top) and MCNPX (Bottom)

The parameterization of these curves is shown in Fig 4a. These curves can be defined by the equation $P(E)=\alpha \exp \left(-\beta E+\gamma E^{2}+\delta \sqrt{E}\right)\left(1-\frac{E}{E_{p}}\right)$ 
where $\alpha, \beta, \gamma$ and $\delta$ varies with the proton energy as shown in Fig $4 \mathrm{~b}$.
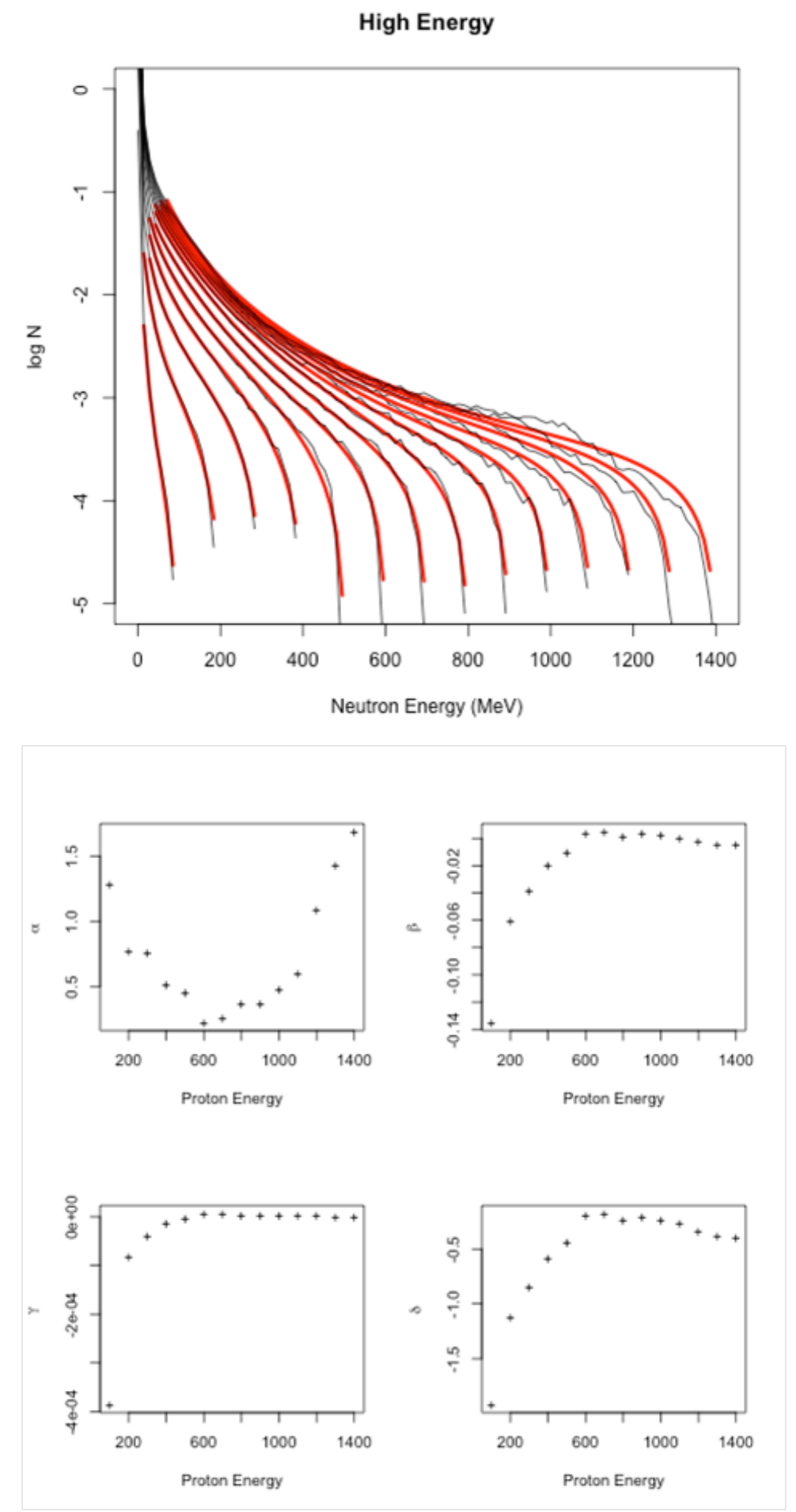

Figure 4: a) High energy parameterisation b) Coefficients $\alpha, \beta, \gamma$ and $\delta$ from left to right 


\subsection{Low energy neutrons}

At low energies (below $1 \mathrm{MeV}$ ) the neutron spectrum is fairly flat, with an intricate shape which is independent of the proton energy, and is similar from each face (Fig 5, Geant4). The low energy neutron could have suffered a number of elastic scatters off the moderator and 'forgotten' their origin. So the spectrum is independent of beam energy. The shape of neutron spectrum at low energies is dominated by cross sections in lead. It can be seen from figure that the spectra from the different faces are identical which is in contrast to the high energy spectra of fig 3 . Further the MCNPX plots in fig 5 reveals that the spectra are identical in shape but differs in magnitude for different proton energies.
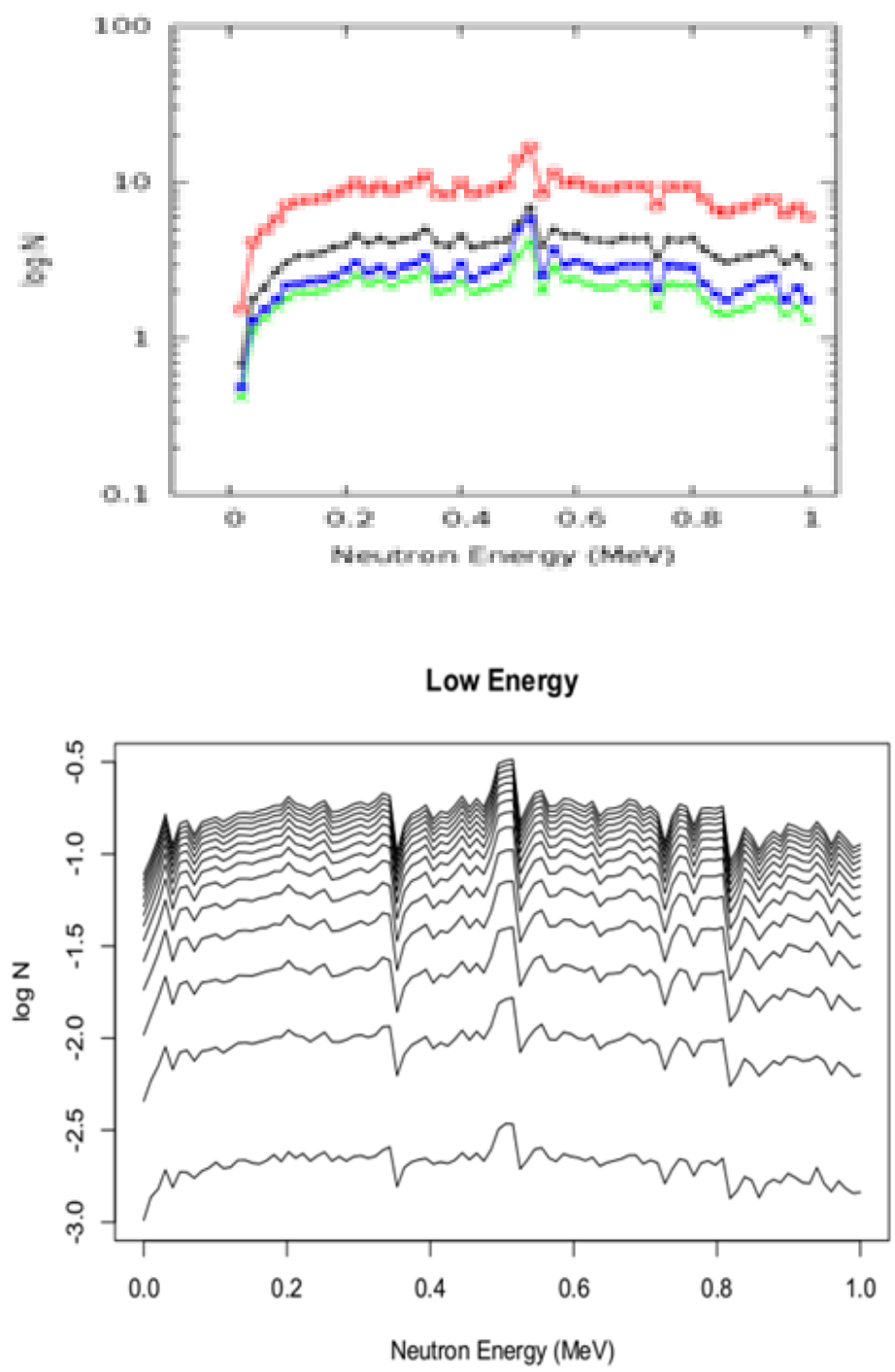

Figure 5: Low energy spectra Geant4 (Top) and MCNPX (Bottom) 


\subsection{Intermediate energy neutrons}

From the above discussion we can say that at low neutron energies the shape of spectrum is independent of proton energy as well as faces and only the size changes and is complicated to parameterize. At high neutron energies the shape of spectrum depends on proton energy and faces and is simple i.e. exponential with cutoff. The question arises "What is high and low?" It is a relative term and it can be defined by looking at the spectra of intermediate energy neutrons (Fig 6 and Fig 7) and the plots says that $\sim 2 \mathrm{MeV}$ is the limit for faces and $\sim 10 \mathrm{MeV}$ is the limit for proton energy dependence. It means that below $2 \mathrm{MeV}$ the spectra is categorized as low (independent of faces) and above $2 \mathrm{MeV}$ (dependent on faces) is high for face dependence. While below $10 \mathrm{MeV}$ the spectra is categorized as low (independent of beam energy) and above $10 \mathrm{MeV}$ (dependent on beam energy) is high for beam energy dependence.
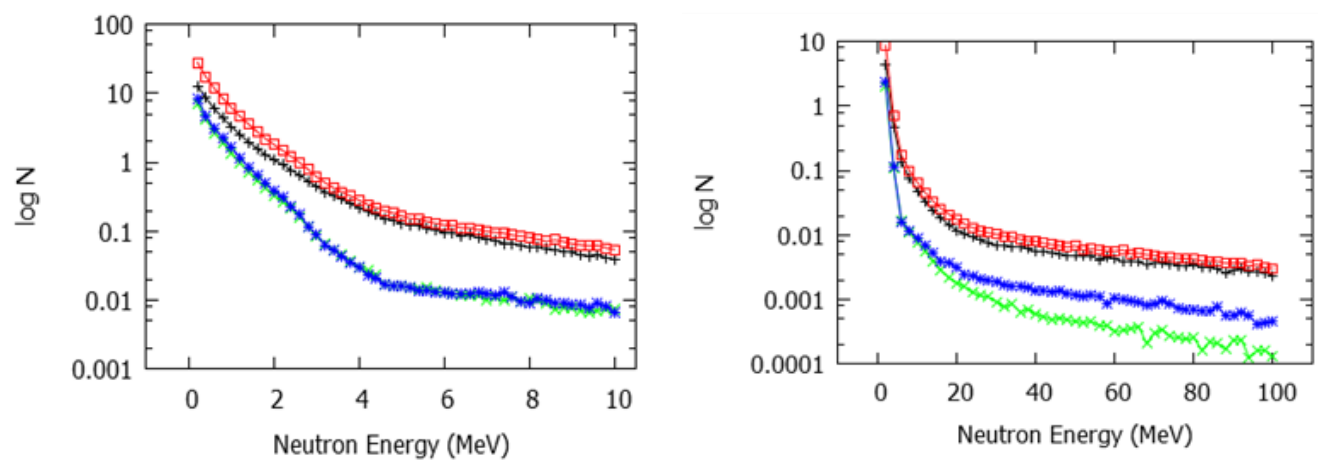

Figure 6: Intermediate energy spectra (Geant4)
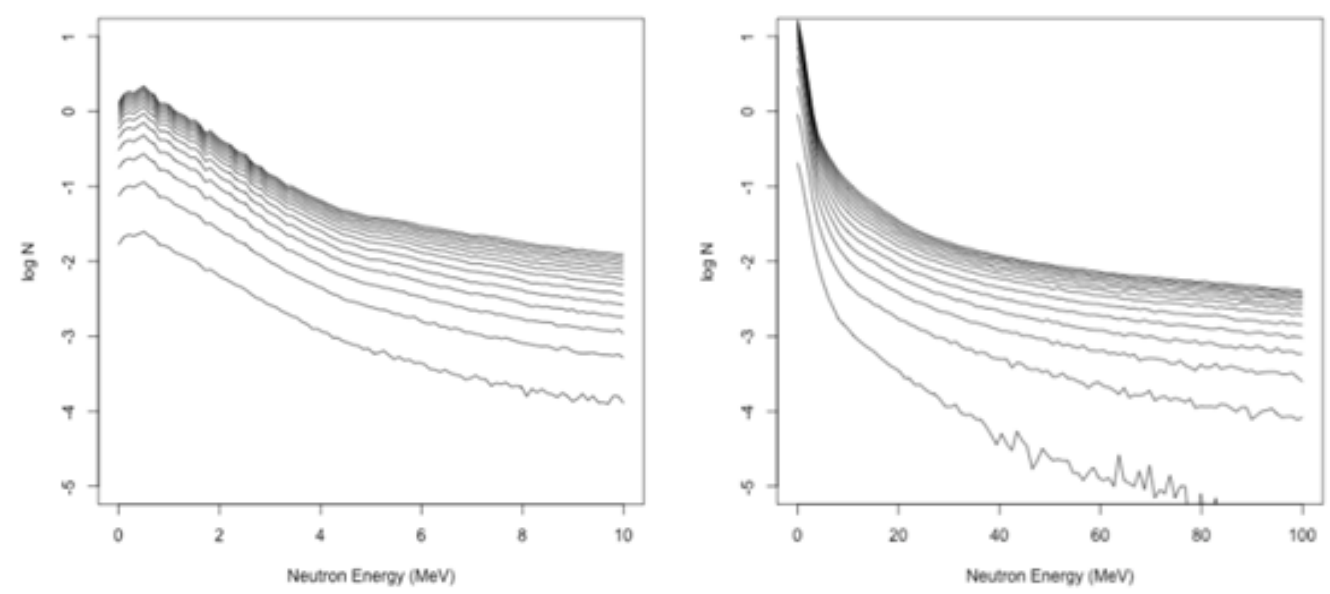

Figure 7: Intermediate energy spectra (MCNPX) 


\section{SPATIAL DISTRIBUTIONS}

\subsection{Longitudinal distributions}

The neutron shower obtained from the longitudinal distribution shows similar behavior for Geant4 and MCNPX (Fig 8). Similar shapes for all proton energies, almost symmetric about mid plane. The plots are normalized to show the number of neutrons/proton $/ \mathrm{cm}$.

For the neutrons emitted along the side, the longitudinal distribution varies remarkably little with energy. It is well described by a parabola. The center of the parabolas increases only very slowly with proton energy.
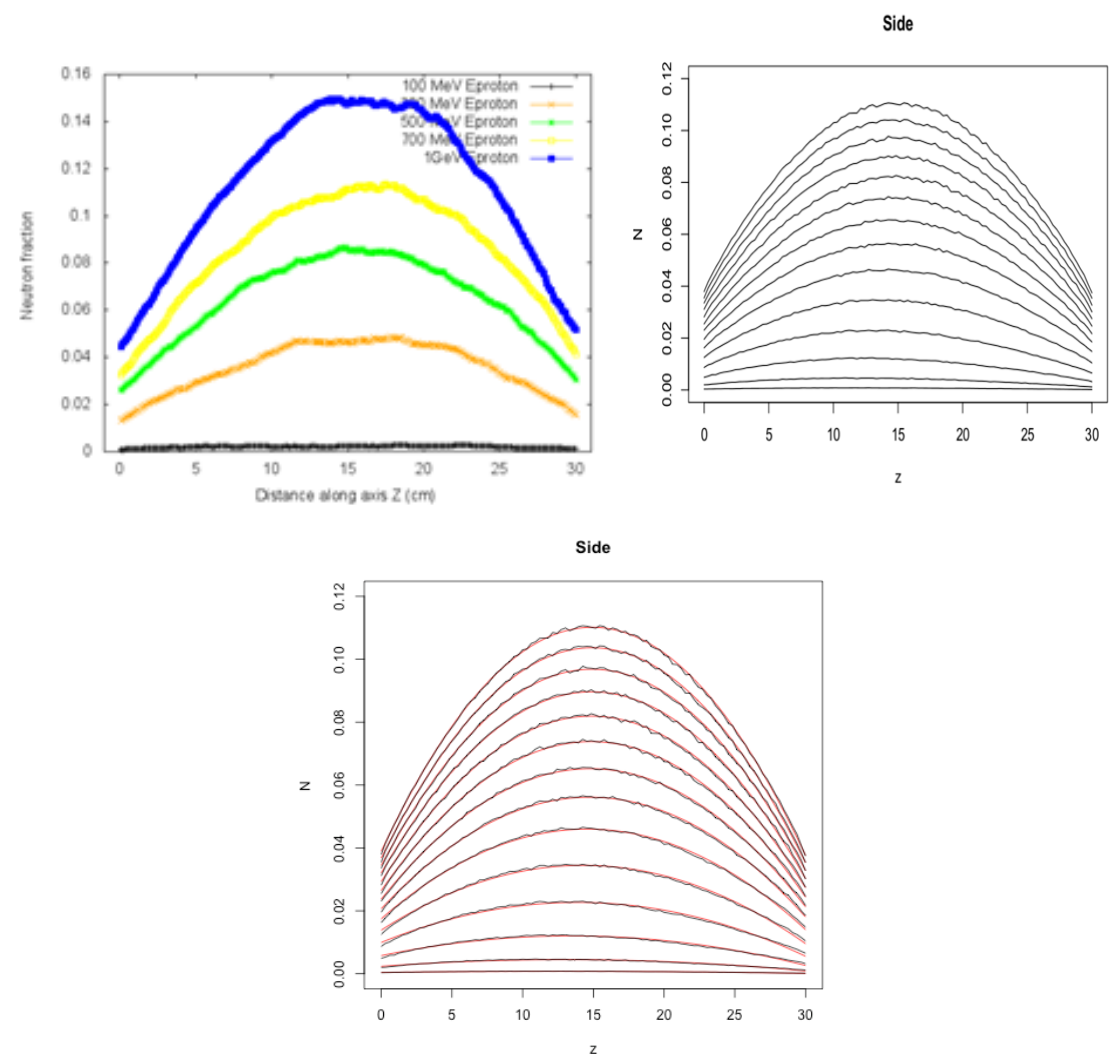

Figure 8: z-distribution Geant4 (Left), MCNPX (Right)and Parameterisation (Bottom)

\subsection{Radial distributions}

The r-distribution of neutrons coming out of the two ends is similar at large $r$ but interestingly different at $r=0$. For the far face the distribution falls to zero at small $r$ while for near it does not. The radial distribution for the two ends obtained by Geant 4 and MCNPX are shown in Fig 9 and Fig 10. The prediction of the two programs agrees well for the two faces. The plots are normalized to show the number of neutrons/proton $/ \mathrm{cm}$. 

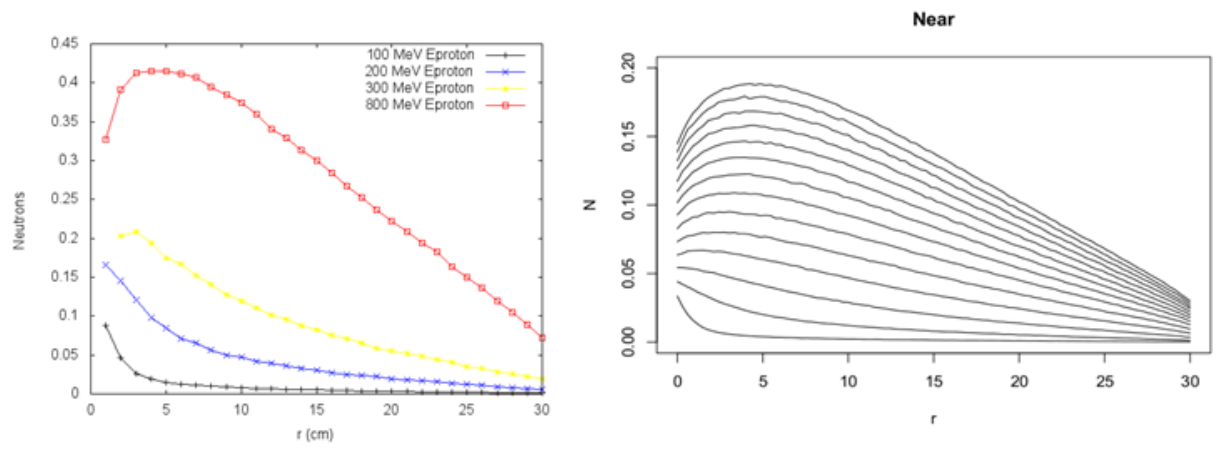

Figure 9: radial distribution from near end Geant4 (Left) and MCNPX (Right)
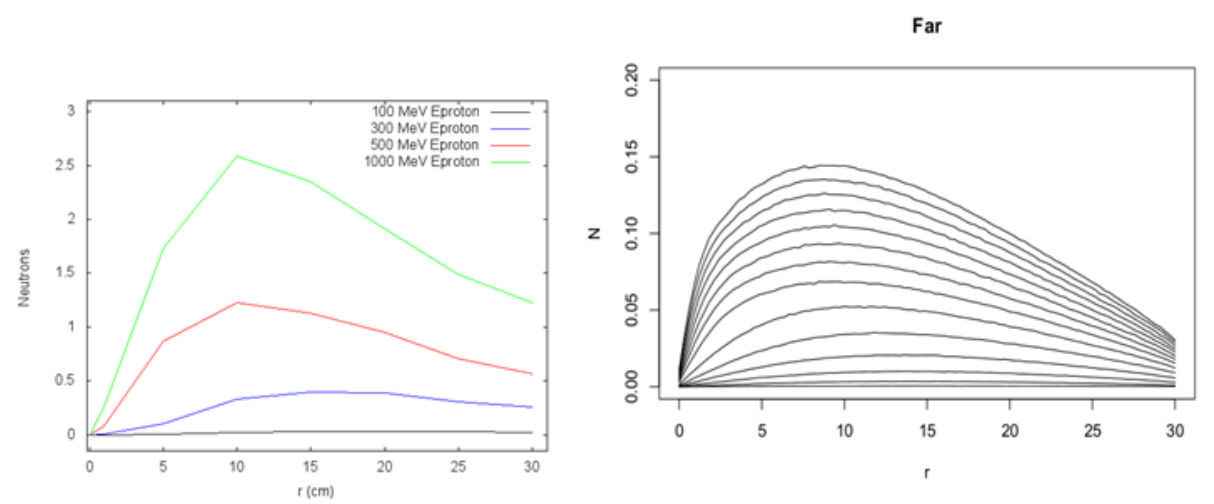

Figure 10: radial distribution from far end Geant4 (Left) and MCNPX (Right)
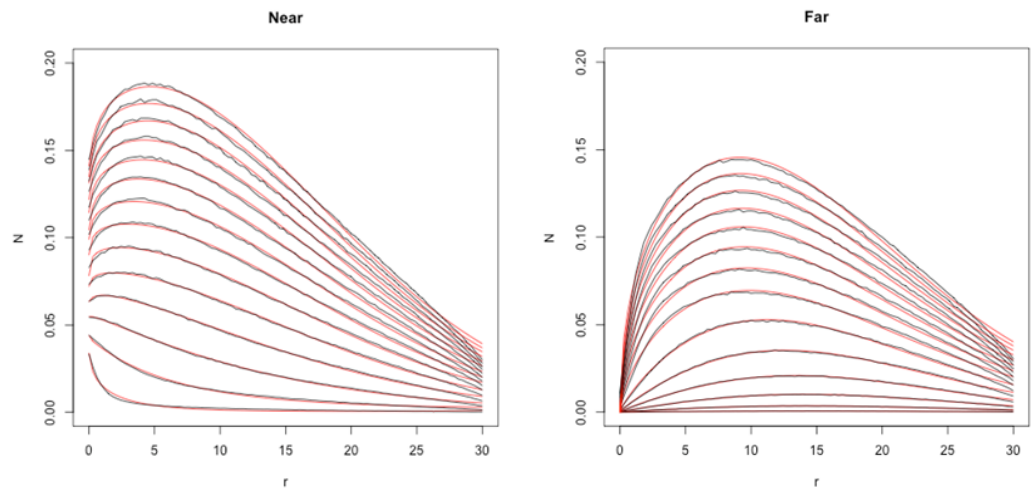

Figure 11: Parameterisation of r-distribution Near (Left) and Far (Right)

The parameterisation of the radial distribution (Fig 11) gives the following equations:

Far face: $P(r) \propto r^{a} \exp \left(-b r-c r^{2}\right)$

Near Face: $P(r) \propto(r-d)^{a} \exp \left(-b r-c r^{2}\right)$ 


\section{DIRECTIONS}

\subsection{Directions in $\theta$}

Fig 12 shows the angular distribution that is the direction made by the cosine of the neutron travel with the z-axis. These are fairly isotropic. From the near face, the particles emerges backwards with a maximum at $\cos \theta=-1$. The distribution is almost linear but also has a quadratic component. Far is a reelected picture of the near plot with the maximum at $\cos \theta=1$. For neutrons coming out of the sides the peak direction is near 90 degrees and the distribution can be described by a quartic. In all the three cases, the parameters vary slowly with the proton energy.
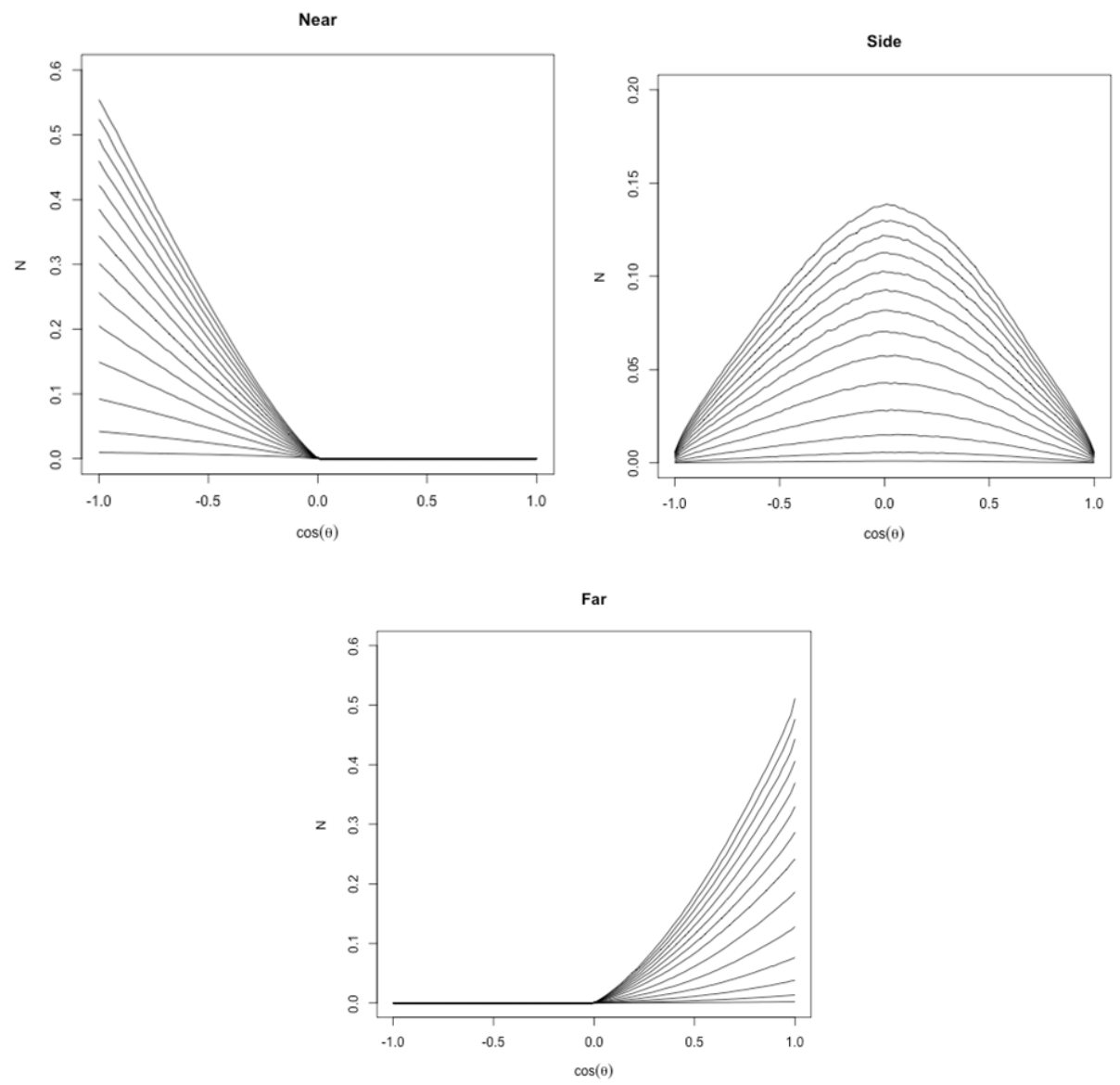

Figure 12: Direction of neutron travel Near (Left), Sides (Right) and Far (Bottom)

\subsection{Directions in $\psi$}

The distribution in angle $\psi$ is shown in Fig13. Angle $\psi$ is the difference between the direction of travel and the azimuth where the neutron emerges. The peak at $\psi=0$ shows the signature of the particle with single scatter. For multiple scattering there is a wide variation in $\psi$ that can be parameterised by a simple polynomial over the possible range of $2 \pi$ for the end faces and $\pi$ for the sides. 

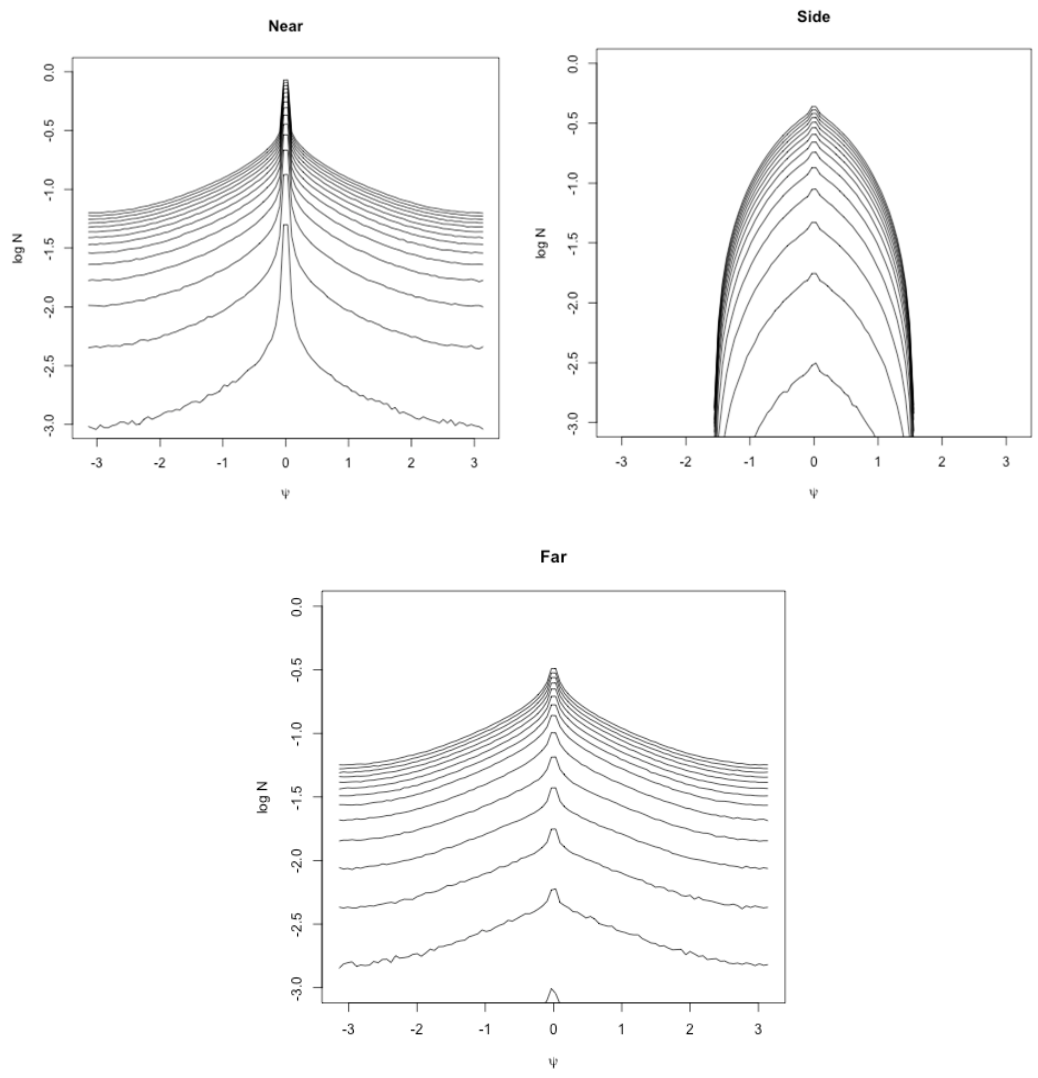

Figure 13: Direction of neutron travel in $\psi$ Near (Left), Sides (Right) and Far (Bottom)

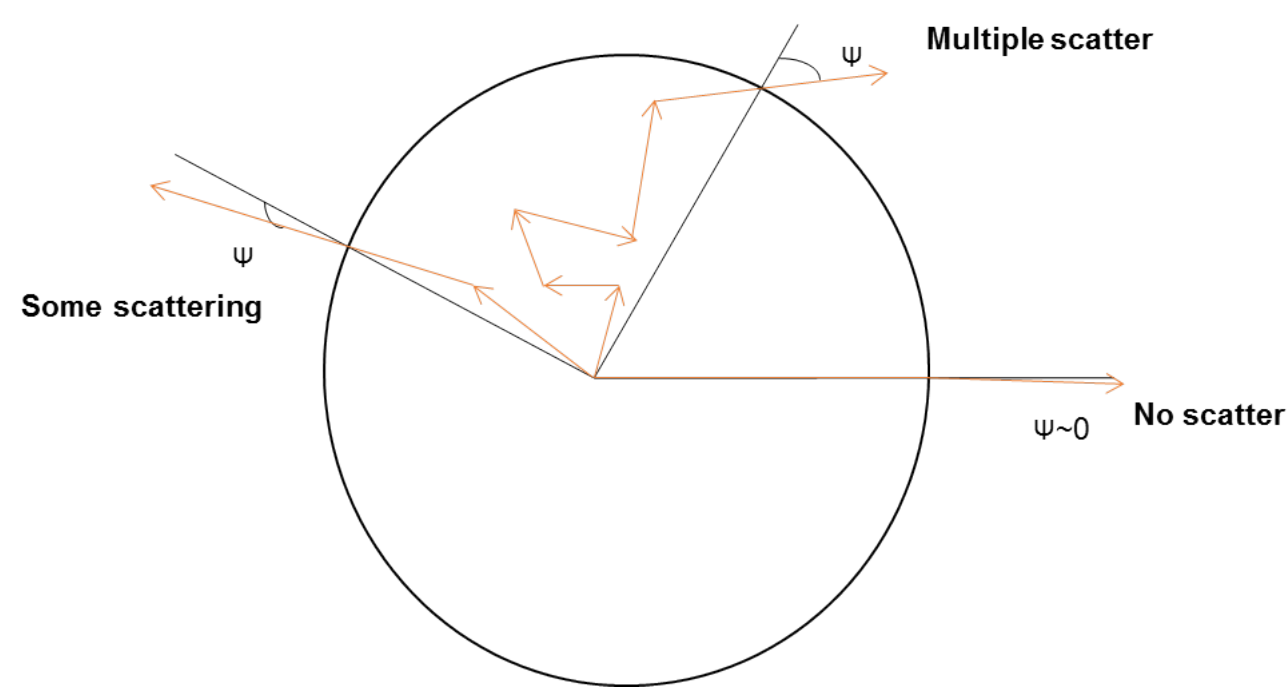

Figure 14: Particle scattering and the azimuth angle 


\section{CROSS SECTIONS AND MODEL DEPENDENCE}
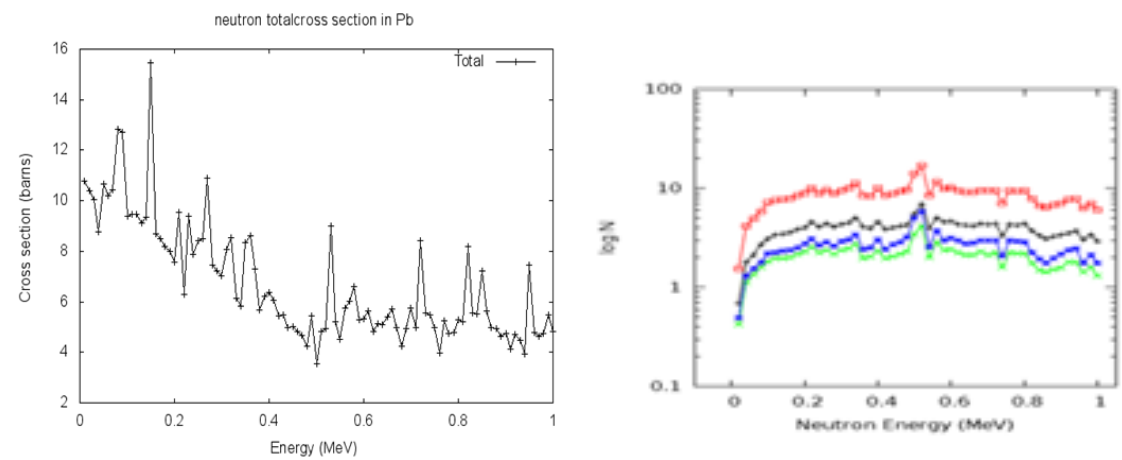

Figure 15: Correlation between crosssection (left in figure) and energy spectra (right)

There is a correlation between the energy spectrum of spallation neutrons and their cross sections in lead. Both the codes predict that the peak in the spectrum matches the dips in the cross section. There is a peak in the neutron spectrum around $500 \mathrm{KeV}$ which corresponds to a minimum of the cross section.

Using Geant4 'out of the box' gave results considerably different from MCNPX: the default option to use models for cross sections is inapplicable for low energy neutrons, where resonances dominate. Hence a High Precision model, using a cross section library, had to be used. It is found that the binary cascade BIC_HP model agreed better with the MCNPX predictions than BERT_HP. For MCNPX ENDF/B-V library [7] is used. Using ENDL92 tables provided in the release gave different cross section that is compared with the cross sections using ENDF/B-V library. This comparison is shown in Fig 16.

ENDF/B-V

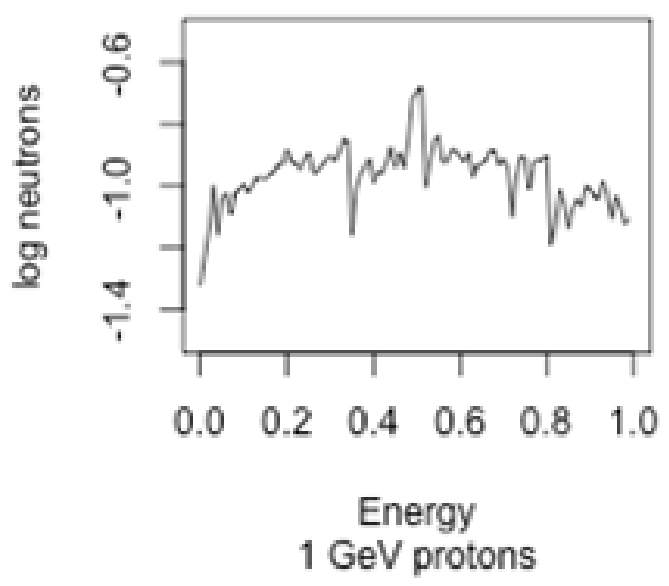

ENDL

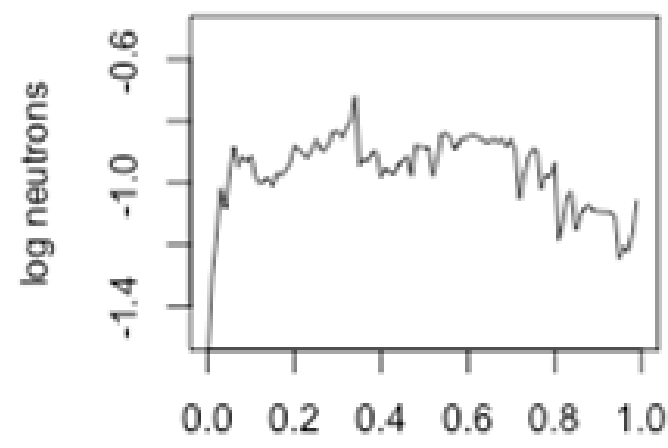

Energy $1 \mathrm{GeV}$ protons

Figure 16: Model dependence of cross sections in MCNPX 


\section{CONCLUSION}

The simulations performed in the study predict that there is roughly a linear rise in neutrons with proton energy with the usual prediction of about 30 neutrons/GeV. Interestingly more neutrons emerge from the near face where the beam hits. The two codes MCNPX and Geant 4 agrees broadly for numbers, energy spectra, positions and directions but differs in details such as relative contribution of the sides and ends for neutrons as well for the low energy spectra (below 1 $\mathrm{MeV}$ ) which is dependent on the use of different cross section libraries. The positions, spectra and the directions are describable by simple parameterizations. These simple forms can be used in the target design and neutronic studies for the reactor cores.

\section{References}

[1] C. Bungau, R. Barlow, A. Bungau, R. Cywinski.Neutron Spallation studies for an accelerator driven subcritical reactor.In proceedings of PAC09, Vancouver, BC, Canada

[2] J.Allison et al. Geant4 Developments and Applications IEEE Trans. Nucl. Sci. 53270 (2006)

[3] D. B. Pelowitz (ed.) The MCNPX user's Manual Version 2.6.0 Los Alamos report LA-CP-07-1473 (2008)

[4] R. Barlow and A. Rummana, Simulation of Neutron Distributions for ADSRs. Proc.12th Int. Meeting on Nuclear .Applications for Accelerators (AccApp'15), Washington DC. To be published

[5] R. Barlow, A. Rummana and R.Seviour (2016). Characterization of the Spectra of Spallation Neutron Sources through Modelling. In 7th International Particle Accelerator Conference (IPAC'16), Busan, Korea, May 8-13, 2016 (pp. 1950-1952). JACOW, Geneva, Switzerland

[6] H. Nifenecker, O. Meplan and S. David, Accelerator Driven Subcritical Reactors IOP publishing (2003)

[7] B. A. Magurno, et al. Guidebook for the ENDF/B-V Nuclear Data Files, EPRI report NP-2510 (1982) 